\title{
THE LUMINOSITY CALIBRATION OF THE HR DIAGRAM
}

\author{
A.E. GÓMEZ ${ }^{1}$, X. LURI ${ }^{1,3}$, M.O. MENNESSIER ${ }^{2}$, J. TORRA $^{3}$ \\ AND \\ F. FIGUERAS ${ }^{3}$, F. ROYER ${ }^{1}$ \\ 1 Observatoire de Paris-Meudon, D.A.S.G.A.L., CNRS URA 335 \\ $P l$. J. Janssen, 92195 Meudon Cedex, France \\ 2 Universite de Montpellier II \\ Pl. E. Bataillon, 34095 Montpellier Cedex 5, France \\ 3 Universitat de Barcelona \\ Diagonal, 647, 08028 Barcelona, Spain
}

An extensive work on the luminosity calibration of the HR diagram has been performed using the LM method based on the Maximum Likelihood estimation (Luri et al. 1996). The method uses all available information: Hipparcos parallax and proper motion data as well as radial velocities and some measured spectroscopic or photometric parameters related to luminosity. On the other hand, the method takes into account the censorship of the sample and the errors of the data. Finally, it is able to identify and separate in a given sample, groups with different luminosity, kinematical or spatial characteristics providing not only the corresponding luminosity calibration relationship, but also improved individual absolute magnitude estimates.

In order to asses how reliable the luminosity calibrations based on the MK spectral classification are, the LM method has been applied to a sample of B to K stars with MK classification selected from the Hipparcos survey. All known binaries, variables and spectroscopically peculiar stars were removed from the sample. The final sample contains 22054 stars. Our results show that the relation between absolute magnitude and luminosity class has a large intrinsic dispersion. As a consequence, the assignation to a star of a mean absolute magnitude given by a MK classification calibration is a rough procedure. It is preferable to use the individual absolute magnitudes estimated using all the available information for each star, like the estimates provided by the LM method (Gómez et al. 1997).

The LM method is well adapted to study the evolutionary state of Barium stars which spread out over a large range in luminosity, from dwarfs to supergiants, most of them showing luminosities of normal giants. From a sample of 297 stars, our results confirm that Ba stars constitute an inhomogeneous group. Five groups were found: supergiants, normal giants, giants concentrated in the clump, dwarfs and a small group of stars with kinematical behaviour of halo stars with a large spread in luminosities. Some of the supergiants could be 'false' Ba stars. Moreover, stars with masses greater than $3 M_{\odot}$ are found in contradiction with current theories for the enhancement of s-process elements in Ba stars (Mennessier et al. 1997).

A Period-Luminosity relation in the $\mathrm{K}$ band has been obtained for Oxygen-rich Mira variables (103 stars). Two main groups has been found corresponding to old disk stars and to a mixing of thick disk and halo stars, respectively. The two PL relations have similar slopes, the shift being probably due to metallicity effect (Alvarez et al. 1997).

\section{References}

Alvarez, R., Mennessier, M.O., Barthès, D., Luri, X., Mattei, J. (1997), A\&A, in press

Gómez, A.E., Luri, X., Mennessier, M.O., Torra, J., Figueras, F. (1997), Hipparcos Venice'97, ESA SP-402, in press

Luri, X., Mennessier, M.O., Figueras, F., Torra, J. (1997), $A \& A S, 117$ pp. 405-415

Mennessier, M.O., Luri, X., Figueras, F., Gómez, A.E., Grenier, S., Torra, J., North, P. (1997), $A \& A$, in press 